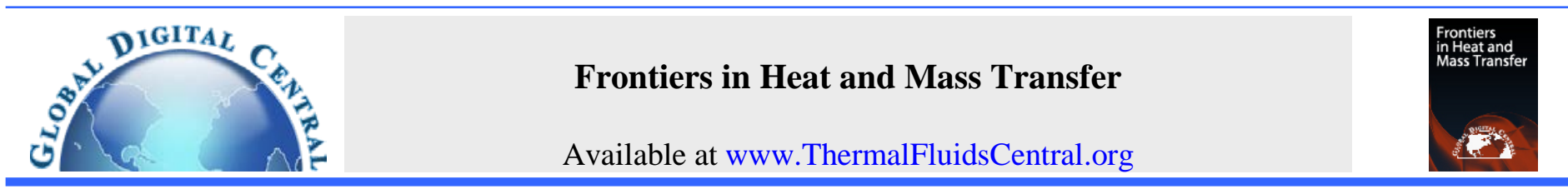

\title{
LONG-TERM ON-ORBIT THERMAL PERFORMANCE OF AEROGEL INSULATION
}

\author{
Kevin W. Irick ${ }^{\mathrm{a}}$, Sally M. Smith ${ }^{\mathrm{b}}$, Andrew D. Williams ${ }^{\mathrm{b}, *}$, Derek W. Hengeveld ${ }^{c}$, Joy M. Stein ${ }^{\mathrm{b}}$ \\ ${ }^{a}$ Applied Technology Associates, Albuquerque, New Mexico, 87123, USA \\ ${ }^{b}$ U.S. Air Force Research Laboratory, Kirtland AFB, New Mexico, 87117, USA \\ ${ }^{c}$ LoadPath, Albuquerque, New Mexico, 87106, USA
}

\begin{abstract}
This paper provides an analysis of the thermal performance of aerogel insulation used during a long-term International Space Station (ISS) flight experiment aboard Space Test Program - Houston 3 (STP-H3). The Variable emissivity device Aerogel insulation blanket, Dual zone thermal control Experiment suite for Responsive space (VADER) investigation tested a variable emissivity radiator and a new form of multi-layer insulation that used aerogel as the thermal isolator. An effort was made to evaluate the performance of the aerogel insulation over the active flight period. The available flight temperature data shows no evidence of deterioration or change in the aerogel insulation's thermal performance during the two-year on-orbit period.

Keywords: radiation, thermal protection, International Space Station
\end{abstract}

\section{INTRODUCTION}

The Variable emissivity device Aerogel insulation blanket, Dual zone thermal control Experiment suite for Responsive space (VADER) was developed by the United States Air Force Research Laboratory (AFRL). In collaboration with the United States Department of Defense Space Test Program (STP), AFRL used VADER as an investigation testbed for a variable emissivity thermal radiator and a new form of multi-layer insulation (MLI). MLI blankets have been the primary form of thermal radiation insultation on spacecraft for decades, and the blankets consist of a alternating layers of highly reflective foils and non-conductive spacer fabric layers. The new, experimental form of MLI on VADER used aerogel as the thermal isolator in order to protect the spacecraft from the harsh extremes of the space environment. The aerogel material provides a more durable, light, and cheaper alternative to traditional spacecraft thermal blankets. The use of this material could reduce the costs related to spaceflight by reducing the required mass and increasing design efficiency.

VADER was launched to the International Space Station (ISS) in May 2011 as part of Space Test Program - Houston 3 (STP-H3) aboard the last spaceflight of the Space Shuttle Endeavor on STS-134. The VADER experiment had two objectives: 1) to measure one-year thermal performance of electrochromic variable emissivity device (VED) panels in the low earth environment, and 2) to measure one-year thermal performance of the silica aerogel insulation. VADER collected aerogel thermal data from June 8, 2011 to August 25, 2013, providing a two-year period for performance comparison.

Figure 1 shows the bare VADER infrastructure prior to component insulation as compared to VADER's on-orbit flight configuration with installed aerogel insulation. VADER was one of four investigations mounted to the STP-H3 platform and was located at the ExPRESS Logistics Carrier site 3 (ELC-3) as an attached, unpressurized payload.
The VADER module assembly was comprised of an aluminum chassis, VEDs and reference samples, and aerogel thermal insulation. The front of the chassis, which contained the VED modules, was left uncovered, while the back of the chassis and brackets were covered with the aerogel insulation, as seen in Fig. 2.

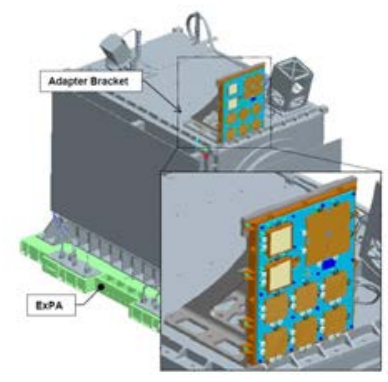

a)

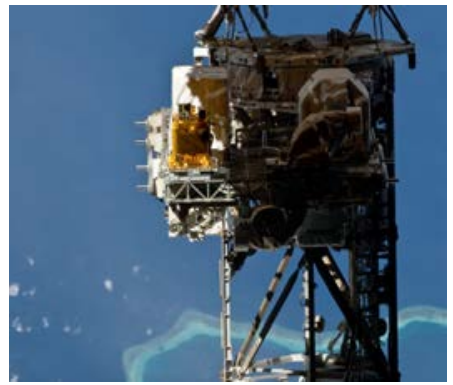

b)
Fig. 1 VADER on STP-H3: a) VADER's location on STP-H3, b) VADER on orbit (Photo Credit: NASA)

The aerogel material, manufactured by Aspen Aerogels ${ }^{\circledR}$, was constructed of the following layup:

Table 1 Insulation layup

\begin{tabular}{|c|c|c|}
\hline \multirow{5}{*}{ Outboard } & Layer & Material \\
\hline & 1 & Kapton, double-aluminized, 1 mil thick \\
\hline & 2 & Mylar, double-aluminized, 1 mil thick \\
\hline & 3 & Aerogel blanket, $3.7 \mathrm{~mm}$ thick \\
\hline & 4 & Mylar, double-aluminized, 1 mil thick \\
\hline & 5 & Kapton, double-aluminized, 1 mil thick \\
\hline
\end{tabular}




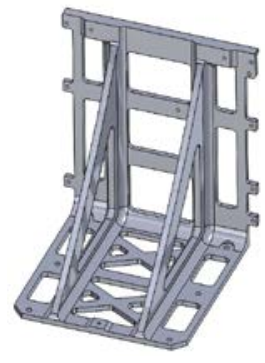

a)

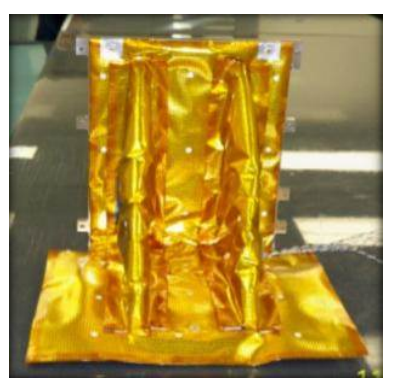

b)
Fig. 2 VADER structure: a) 3D model of aluminum chassis, b) flight structure with aerogel insulation

\section{BACKGROUND}

Spacecraft are subject to a number of potentially hazardous environmental conditions that require great levels of consideration in the design stages of their systems and structures. Harsh aerospace conditions can include the presence of atomic oxygen (AO), solar winds, ultra-violet (UV) or x-ray exposure, thermal cycling, space debris, micro-meteorites, rocket-engine plume impingement, and a host of other destructive elements (Finckenor and Dooling, 1999; Gilmore, 2002). Insulation — often covering a major portion of a space structure's exterior-can be the first line of defense against such a destructive environment, and, thus, special considerations must be made with respect to fabrication, venting, electrical grounding, fastening, material orientation, durability, flammability, and launch loads, to name a few (Gilmore, 2002).

AO can be especially hazardous to spacecraft insulation while on orbit. When certain materials are exposed to AO, chemical reactions at the contact surface can form volatile oxides that essentially evaporate into space. Likewise, the AO can form oxides with the insulation material that easily spall and flake, destroying the integrity of the insulation (de Rooij, 2010). Similarly, UV exposure can change both the optical and mechanical properties of some materials, causing degradation and overall destruction of the insulation. UV effects can even change when combined with AO exposure or other variable conditions (Finckenor and Dooling, 1999).

The traditional form of aerospace insulation is multilayer insulation (MLI), a series of reflective thin-films or foils in a blanketlike layup, as shown in Fig. 3, that reduces overall transmission of radiant thermal energy.

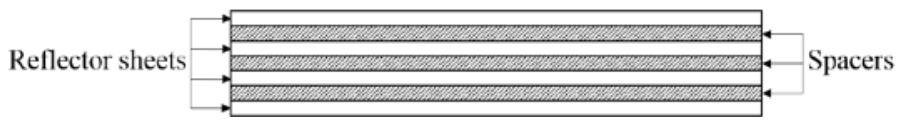

Fig. 3 MLI schematic

An infinite variety of MLI exists, depending on reflector and spacer materials, layer count, reflector and spacer thicknesses, fabrication processes, and overall geometry. Some typical reflector sheet materials include Kapton, Mylar, or even Teflon, and separator materials may include Dacron or Nomex. Both reflector sheets and separators may have thicknesses on the order of 0.1 to 1.0 mil (Finckenor and Dooling, 1999). Reflector sheets may also be aluminized or goldized with or without perforations. In the mid-1900's, multilayer insulation had been investigated for use in spacecraft propulsion and propellant modules (Perkins et al., 1968) and has since been commonly used in countless aerospace operations ranging from private to military space missions on various space vehicles.

A possibly attractive form of alternative aerospace insulation is aerogel. Aerogel are "gels in which the liquid has been replaced by air, with very moderate shrinkage of the solid network" (Aegerter, et al., 2011). There is a variety of forms of aerogel, with a wide range of material properties. Significant changes in material performance can be seen with respect to temperature, gas pressure, density, and porosity of a given specimen. For example, density can range from $5-200 \mathrm{mg} / \mathrm{cm}^{3}$, stiffness can range from $10^{4}-10^{8} \mathrm{~N} / \mathrm{m}^{2}$, and thermal conductivity can span 0.001-0.100 W/m-K (Shang and Zhao, 2012; Aegerter, et al., 2011). These low density and thermal conductivity values make aerogels effective insulators.

Historically, aerogel has been used in a variety of applications such as in aerospace, aeronautical, metallurgical, structural, petroleum, refrigeration, energy, chemical, and biomedical fields (Aegerter, et al., 2011). More specifically, in aerospace, aerogel has been used for orbital debris and space dust collection and general spacecraft thermal insulation-including as thermal insulation on a Mars rover (Shang and Zhao, 2012; Aegerter, et al., 2011).

The last set of on-orbit aerogel thermal data collected by AFRL was made on August 25 2013. Aerogel has since been evaluated inhouse as a radiative barrier using a guarded hot plate method (Irick, 2017) which shows performance comparable to a range of 5-layer to 10-layer MLI blankets. The ground testing results, along with the durability, low cost of handling and integration, and low density of aerogel, make aerogel a competitive alternative to traditional MLI as an aerospace thermal insulator.

Aerogel as an aerospace thermal radiation barrier lacks opensource performance data. Provided the competitive material properties of aerogel, as compared to MLI, it is useful to empirically assess the performance of the insulation in a spaceflight environment. Likewise, due to the unpredictability and harshness of space, it is necessary for potential users of aerogel to evaluate the reliability and resilience of the insulation in such an environment. This must be done in part to evaluate effects of long-term exposure to the space environment and to understand possible degradation, alteration, or any other change that can affect the insulation's performance.

\section{EVALUATION METHOD}

\subsection{Typical Insulation Evaluation}

Typically, a radiative barrier might be characterized and evaluated by either its thermal conductivity (characterizing mostly its performance as a conductive barrier) or its effective emittance, $\varepsilon^{*}$, where

$\varepsilon^{*}=k_{e f f} \cdot\left(T_{h}-T_{c}\right) /\left[\sigma \cdot t \cdot\left(T_{h}^{4}-T_{C}^{4}\right)\right],(1)$

$k_{\text {eff }}$ is the effective thermal conductivity, $T_{h}$ and $T_{c}$ are the hot and cold boundary temperatures, respectively, $\sigma$ is the Stefan-Boltzmann constant, and $t$ is the insulation thickness (Gilmore, 2002). Effective emittance measures the blanket's performance as a radiative barrier and is the attribute of choice in evaluating aerospace thermal radiation insulation.

\subsection{Spaceflight Data}

Temperature data was collected on orbit in several locations on the VADER structure. A total of 21 temperature sensors were installed on the insulated structure at seven different measurement locations. Figure 4 labels the seven measurement locations. Four of the locations were on the upright section of the chassis, opposite the structural face with VED modules, and the other three locations are found on the base, between the support brackets. At each measurement location, three throughthickness temperature sensors were fixed, as indicated in the following diagram.

Sensors from various locations were routed through various electronics hubs. During flight, data from sensor locations 1, 3, and 5 through 7 were either lost or corrupted, leaving only data from locations 2 and 4 to be analyzed. In the beginning of VADER's flight, temperature data was collected at $1.00 \mathrm{~Hz}$. However, throughout the program, data collection rates alternated between to $0.017,0.10$, and 
1.00 Hz. Despite an order of magnitude decrease in data sampling, the capture of the system's slow response to temperature shifts in the system is not significantly affected by the lower sampling rates. Figure 6 shows how data from each of the three rates compare in resolution.

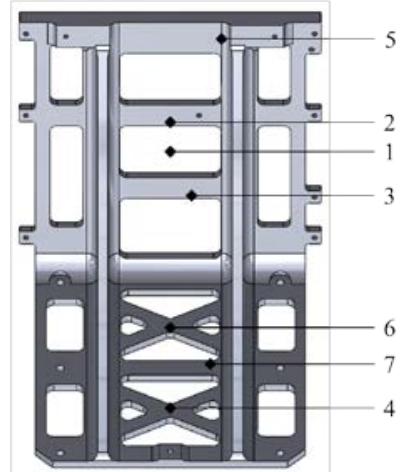

Fig. 4 Temperature measurement locations

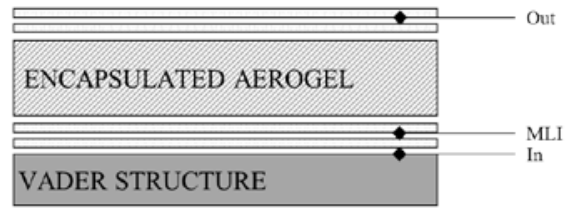

Fig. 5 Through-thickness temperature sensor placement

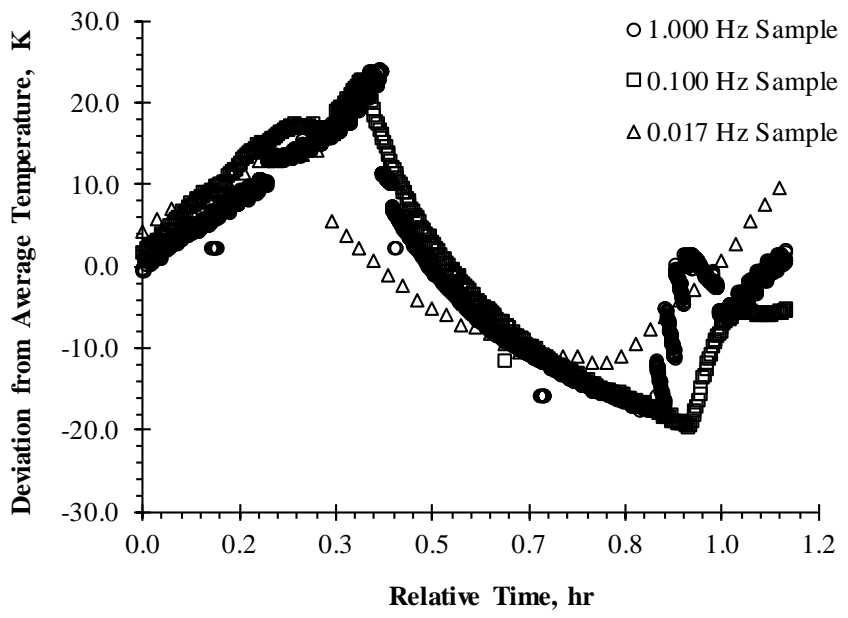

Fig. 6 Data sampling resolution comparison

\section{ANALYSIS APPROACH}

With no knowledge of heat flux incident on the structure insulation, any true material property approximations for the insulation would be extremely difficult to back out (and unreliably so). A variety of failed analysis approaches-including numerical simulations and transient response correlations-proved the limitations posed by the available data and the system's deviation from standard academic scenarios of predictable boundary conditions

It was determined that to make an approximated evaluation of the performance of the insulation over time, a number of assumptions would be made, and the effective emittance of the blanket would be evaluated. Recalling the equation for effective emittance, the following are assumed in this analysis:

1. The effective thermal conductivity of the blanket remains constant relative to both temperature and time. This assumption can be considered appropriate for this application because the average blanket temperature for a given analysis set remains within a $20 \mathrm{~K}$ band.

2. The thickness of the insulation remains constant over time. This assumption is considered to be valid since the aerogel is essentially protected and would not experience any structural stress.

3. Effective emittance can be represented during transience using the traditional calculation, shown in Eq. (1). This assumption is especially valid for a system of pure radiative heat transfer and is expected to be valid for this application due to the high level of porosity-and low density-of the conducting aerogel material.

If these assumptions are made, the only variability left is the ratio of temperature differences, defined now as

$r_{\Delta T}=\left(T_{h}-T_{c}\right) /\left(T_{h}^{4}-T_{c}^{4}\right)$.

The identified assumptions imply that a change in the ratio of temperature differences is directly proportional to and representative of the change in the effective emittance.

\section{RESULTS}

Two analyses were performed on the ratio of temperature differences. In one analysis, the ratio was inspected using data from a full 24-hour day. In another analysis, the ratio was evaluated using only the temperatures where the external temperature reached local maxima during the day. Figure 7 shows an example of the data used for the second method. In the figure, complete time series temperature data is shown for insulation interior and exterior temperatures for a full 24hour period. Bands are shown that are intended to capture data near local exterior temperature maxima throughout the data set. Thus, the red markers indicate data points at which the exterior temperature measurement is considered to be in the region of a local maximum. The red-marked data points are then used in the analysis approach only using the local maxima set.

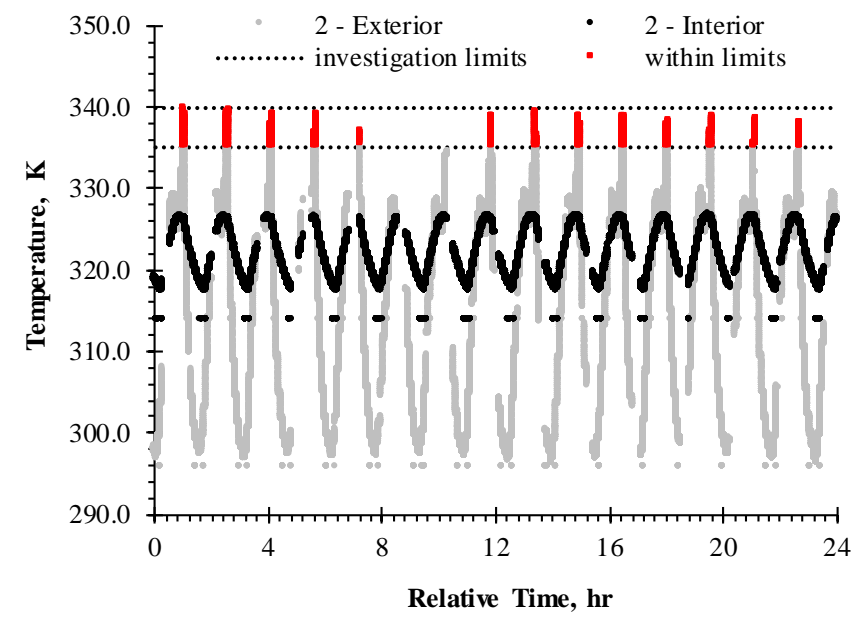

Fig. 7 Local maxima investigation window

Instead of computing a single ratio value based on average temperatures from a given data set, a ratio was computed at each sample point during the day. For each data set, an average ratio was then computed, as show in Eq. (3), where $N$ is the total number of data points in a given data set.

$r_{\Delta T}=\sum_{i=1}^{N}\left[\left(T_{h, i}-T_{c, i}\right) /\left(T_{h, i}^{4}-T_{c, i}^{4}\right)\right] / N$ 
For an object in orbit, the beta angle of an orbit represents the angle between the orbital plane and the sun vector. Thus, the beta angle can have a significant impact on the solar irradiation to the object in orbit. In an attempt to compare days of probable similar incident heat flux conditions for this study, five pairs of days were used in which the beta angles for the days in each pair were similar. Table 2 lists the comparison cases.

Table 2 Evaluation comparison cases

\begin{tabular}{|c|c|c|c|}
\hline Comparison & \multicolumn{2}{|c|}{ Date } & Beta Angle \\
\hline \multirow{2}{*}{1} & A & December 18, 2012 & $3.4^{\circ}$ \\
\cline { 2 - 4 } & B & August 21, 2013 & $3.7^{\circ}$ \\
\hline \multirow{2}{*}{2} & A & August 20, 2012 & $22.8^{\circ}$ \\
\cline { 2 - 4 } & B & August 18, 2013 & $22.8^{\circ}$ \\
\hline \multirow{2}{*}{3} & A & August 19, 2012 & $27.6^{\circ}$ \\
\cline { 2 - 4 } & B & August 16, 2013 & $27.6^{\circ}$ \\
\hline \multirow{2}{*}{4} & A & August 18, 2012 & $32.4^{\circ}$ \\
\cline { 2 - 4 } & B & August 15, 2013 & $32.4^{\circ}$ \\
\hline \multirow{2}{*}{5} & A & June 9, 2011 & $45.9^{\circ}$ \\
\cline { 2 - 4 } & B & August 12, 2013 & $46.4^{\circ}$ \\
\hline
\end{tabular}

The ratios of temperature differences for each comparison using the full day analysis and the local maxima analysis are shown in Fig. 8 and Fig. 9, respectively. Here, " 2 " and "4" refer to the temperature measurement locations, and " $\mathrm{A}$ " and " $\mathrm{B}$ " denote the earlier and later dates in the comparison set, respectively. The results shown in both Fig. 8 and Fig. 9 show that the ratios of temperature differences at each comparison are statistically similar, with the exception of comparison 1 in Fig. 9, implying no quantified change in performance over time.

Of note is the distinguishable behavioral trend of the temperature difference ratios with respect to beta angle, regardless of time. Figure 10 shows the beta angle for each day over a representative year of the experimental data set, illustrating the variation in incident solar heat flux. Figure 11 and Fig. 12 show how the temperature difference ratios change with respect to beta angle, each decreasing with increasing beta angle, indicating the change in incident heat flux on the insulation. This change directly changes the temperature differential between hot and cold surfaces and, therefore, the effective emittance.

Because temperature ratios are computed at each sample point, the error bars in the charts of Fig. 8 and Fig. 9 are based solely on the standard deviation of the temperature difference ratio calculations and not on measured values. Therefore, the uncertainty represents a minimum uncertainty approximation.

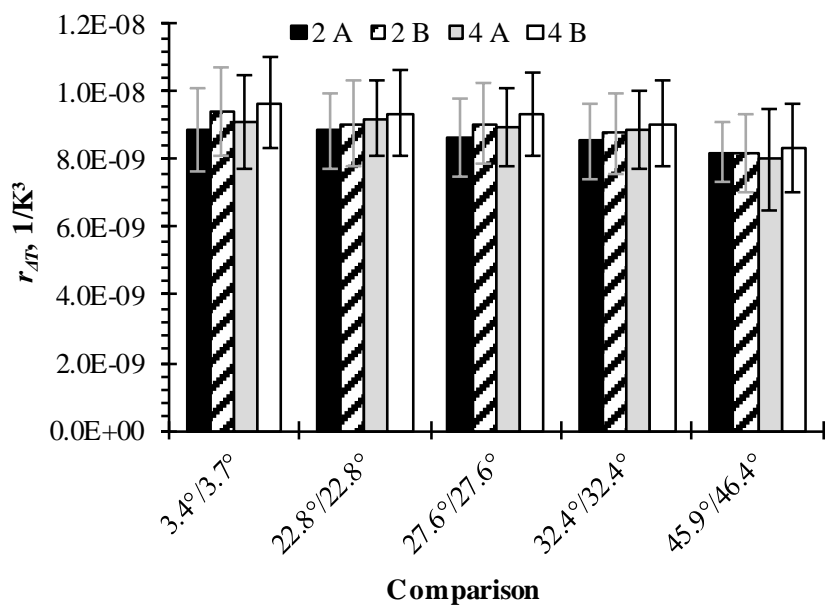

Fig. 8 Temperature difference ratio full day data set comparisons

In all of the full day comparisons, the average temperature difference ratios exhibit no statistically distinguishable difference. Likewise, the same is shown in four of the five comparisons using the local maxima approach. If a more thorough uncertainty analysis were possible, it is likely that every comparison would show no distinct difference. Therefore, the results_-as seen in these charts-suggest that no conclusive change is observed in the performance of the aerogel insulation over time during flight.

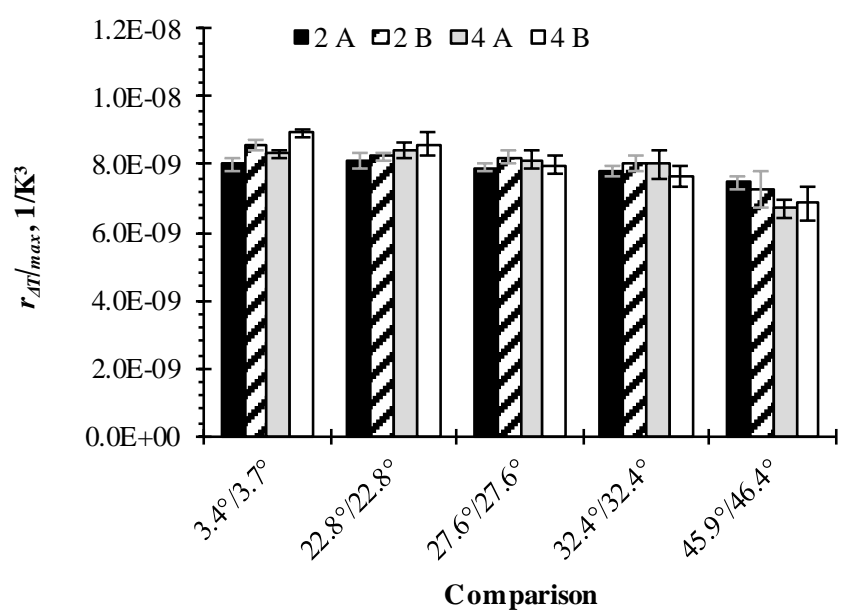

Fig. 9 Temperature difference ratio local maxima data set comparisons

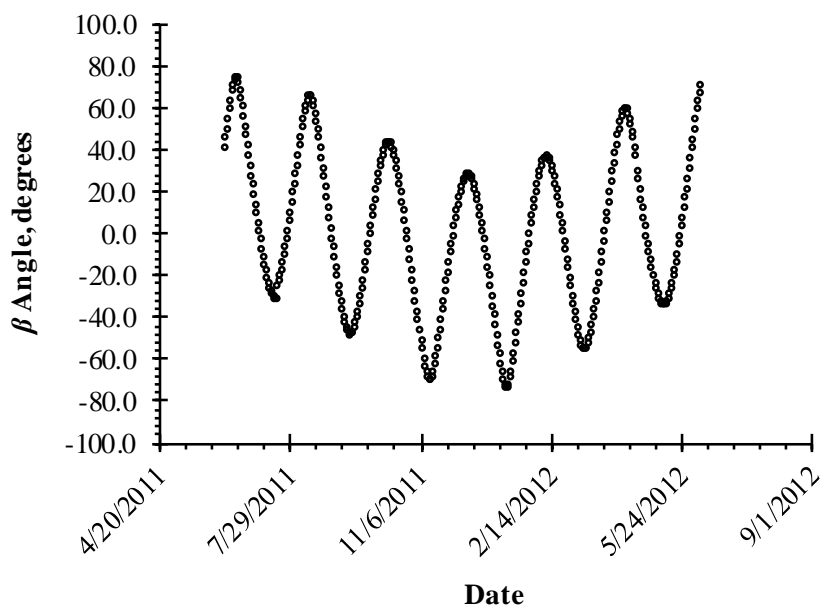

Fig. 10 Beta angle vs. date

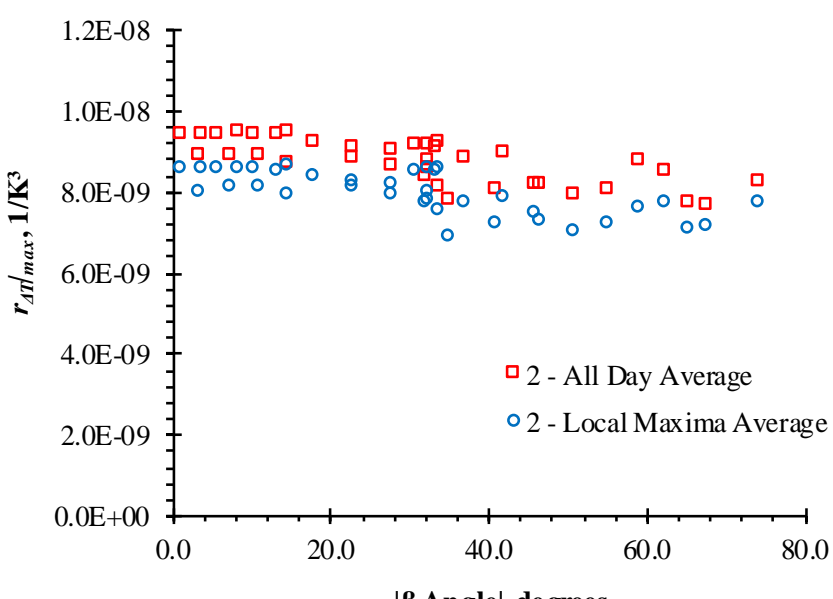

| $\beta$ Angle|, degrees

Fig. 11 Temperature measurement location 2 temperature difference ratio vs. beta angle 


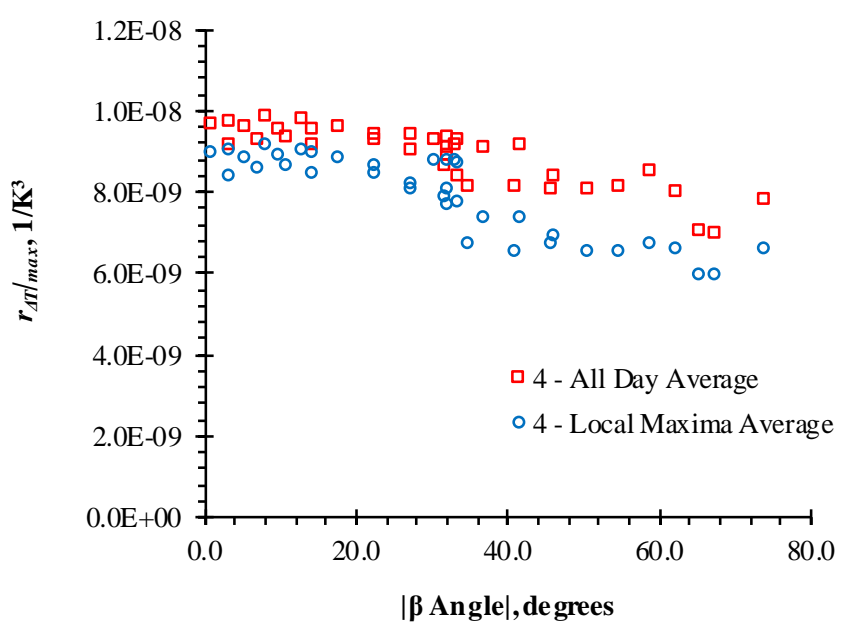

Fig. 12 Temperature measurement location 4 temperature difference ratio vs. beta angle

\section{CONCLUSIONS}

With emphasis on the assumptions made, it is concluded that between June 8, 2011 and August 25, 2013 the VADER aerogel insulation experienced no significant change in performance as a radiative barrier during space flight. In ongoing efforts to assess the resiliency and performance of next-generation space structures, the results provided by the VADER aerogel thermal data shed promising light on alternative aerospace insulation options.

One of the lessons learned from this research effort is that a precise and accurate evaluation of thermal radiation barriers is complex, especially when the analysis is done remotely to transient, on-orbit systems. Therefore, analysis and evaluation methods should be designed as simply as is feasible. Also, an end-to-end evaluation of the data to be collected could result in more effective instrumentation and data. For example, the addition of heat flux sensors could provide more information about the thermal environment.

The available data and assumptions provide useful insight and promise regarding the long-term performance of on-orbit aerogel insulation blankets.

\section{ACKNOWLEDGEMENTS}

The authors would like to thank Aspen Aerogels ${ }^{\circledR}$ and the U.S. Department of Defense Space Test Program.

\section{NOMENCLATURE}

i summation index

$k_{\text {eff }} \quad$ effective thermal conductivity (W/m-K)

$N \quad$ total number of data points

$q^{\prime \prime} \quad$ heat flux $\left(\mathrm{W} / \mathrm{m}^{2}\right)$

$r_{\Delta T} \quad$ ratio of temperature differences $\left(1 / \mathrm{K}^{3}\right)$

$t \quad$ thickness (m)

$T_{c} \quad$ cold boundary temperature (K)

$T_{h} \quad$ hot boundary temperature (K)

Greek Symbols

$\beta \quad$ beta angle (degrees)

$\varepsilon^{*} \quad$ effective emittance

$\sigma \quad$ Stefan-Boltzmann constant, $5.67 \times 10^{-8} \mathrm{~W} / \mathrm{m}^{2} \cdot \mathrm{K}^{4}$

\section{REFERENCES}

Aegerter, M.A., Leventis, N., Koebel, M.M., 2011, Aerogels Handbook, Springer, New York, NY.

https://doi.org/10.1007/978-1-4419-7589-8

de Rooij, A., 2010, “Corrosion In Space”, Encyclopedia of Aerospace Engineering, John Wiley \& Sons.

https://doi.org/10.1002/9780470686652.eae242

Finckenor, M.M., Dooling, D., 1999, “Multilayer Insulation Material Guidelines”, National Aeronautics and Space Administration, Marshall Space Flight Center, AL.

Gilmore, D.E., 2002, Spacecraft Thermal Control Handbook, Vol. 1, 2nd ed., The Aerospace Press, El Segundo, CA.

Irick, K.W., 2017, "Effective Thermal Resistance Comparison of Aerogel and Multi-layer Insulation as Radiative Barriers Using the Single-sided Guarded Hot Plate Method," Frontiers in Heat and Mass Transfer, (8)2. https://doi.org/10.5098/hmt.8.2

Perkins, P.J., Dengler, R.P., Niendorf, L.R., Nies, G.E., 1968, "Selfevacuated Multilayer Insulation of Lightweight Prefabricated Panels for Cryogenic Storage Tanks”, National Aeronautics and Space Administration, Washington, D.C.

Shang, S., Zhao, D., 2012, Aerospace Materials Handbook, CRC Press, Boca Raton, FL.

https://doi.org/10.1201/b13044 